\title{
Diagnóstico y localización de insulinomas mediante test de estimulación con calcio por cateterismo arterial selectivo
}

\author{
Diagnosis and localization of insulinomas through stimulation test with \\ calcium by selective arterial catheterization. Report of two cases
}

Eduardo LLahyah¹, Esteban Mendaro², Raúl LLano ${ }^{3}$

\section{RESUMEN}

El insulinoma es el tumor neuroendocrino más común, con una incidencia aproximada de 1 a 4 por cada millón de habitantes/año. Típicamente se presenta como un tumor solitario, pequeño, de menos de $2 \mathrm{~cm}$, por lo que su localización preoperatoria suele ser difícil. Además puede aparecer de forma multifocal en el contexto de una neoplasia endocrina múltiple tipo 1 o como un tumor metastásico en el insulinoma maligno. Sus manifestaciones clínicas son frecuentemente confundidas con síntomas neuropsiquiátricos, y el diagnóstico definitivo puede demorarse durante mucho tiempo. Presentamos dos pacientes de distintos centros de atención, con diagnóstico clínico de insulinoma, en los que el estudio de imagen preoperatorio fue negativo. Ambos cumplían criterios diagnósticos, con altos niveles de insulina sérica en presencia de una glucemia menor de $45 \mathrm{mg} / \mathrm{dl}$.

Los estudios de imágenes, incluyendo la tomografía computarizada abdominal y la resonancia magnética, no revelaron la localización del tumor en uno de los pacientes. En el otro se realizó angiografía pancreática con estimulación selectiva con calcio intraarterial y muestreo venoso para mediciones de insulina. Esta prueba permitió la localización exacta de los tumores y su extirpación exitosa.

Palabras claves: test de estimulación intraarterial con calcio; angiografia; insulinoma; tumor neuroendocrino.

\begin{abstract}
Insulinoma is the most common neuroendocrine tumor. Usually, its clinica presentation is as a small, solitary tumour, less than $2 \mathrm{~cm}$ long, which make it difficult to find before surgery. It may also appear multifocally in the context of multiple endocrine neoplasia type 1 or as a metastatic tumor in malignant insulinoma.

Its clinical manifestations are frequently mistaken with neuropsychiatric symptoms, so definitive diagnosis can be delayed for a long time.

We report two patients from different care centers, with a clinical diagnosis of insulinoma, in whom the preoperative imaging study was negative. Both fulfilled diagnostic criteria, with high serum insulin levels in the presence of a blood glucose lower than $45 \mathrm{mg} / \mathrm{dl}$.

A pancreatic angiography with selective stimulation with intra-arterial calcium and venous sampling for insulin measurements, was performed in both patients. This test allowed the exact localization of the tumors and their successful excision.
\end{abstract}

Key words: intra-arterial stimulation test with calcium; angiography; insulinoma; neuroendocrine tumor.

Revista Argentina de Cardioangiología Intervencionista 2018;9(3):167-170. Doi: 10.30567/RACI/20183/0167-0170

\section{INTRODUCCIÓN}

Los insulinomas son tumores endocrinos con una incidencia estimada de 4 casos por millón de habitantes y año ${ }^{1}$. Suelen ser tumores benignos de pequeño tamaño ( $90 \%$ son $<2 \mathrm{~cm})$.

Los criterios diagnósticos necesarios y suficientes para sospechar un insulinoma incluyen: hipoglicemia espontánea inferior a $45 \mathrm{mg} / \mathrm{dl}$, insulinemia mayor a $6 \mu \mathrm{U} / \mathrm{ml}$, péptido $\mathrm{C}$ mayor de $0,2 \mathrm{mmol} / \mathrm{l}$ tomados durante un episodio de hipoglicemia espontánea o durante un test de ayuno y negatividad en el rastreo de sulfonilureas en orina. Una vez confirmada la sospecha de insulinoma, se debe abordar la difícil tarea de localizar anatómicamente el tumor.

El tratamiento de primera elección es la resección quirúrgica, con una eficacia superior al 90\%, y la localización preoperatoria incrementa la eficacia y reduce la morbimortalidad asociada a la cirugía.

Debido a su tamaño y características radiológicas, los insu-

1. Servicio de Hemodinamia. Hospital Privado de Rosario. Rosario, Rep. Argentina.

2. Jefe de Servicio de Hemodinamia. Hospital Naval Pedro Mallo. Investigaciones Vasculares. CABA, Rep. Argentina.

3. Servicio de Hemodinamia. Hospital Naval Pedro Mallo. CABA, Rep. Argentina.

$\triangle$ Correspondencia:Dr.Eduardo Augusto LLahyah.Email:eduardo.Ilahyah@ gmail.com

Los autores no declaran conflictos de intereses

Recibido: 12/10/2017| Aceptado: 24/05/2018 linomas son tumores de difícil localización con las técnicas convencionales $^{2}$ (tomografía computarizada [TC] y resonancia magnética nuclear [RM]), y la ultrasonografía endoscópica puede no detectar el tumor, especialmente cuando está situado en cola de páncreas ${ }^{3}$. Por ello, cuando las técnicas morfológicas no han resultado de utilidad, la localización preoperatoria del insulinoma requiere una técnica funcional realizada mediante radiología intervencionista.

El páncreas está irrigado por las arterias gastroduodenal,

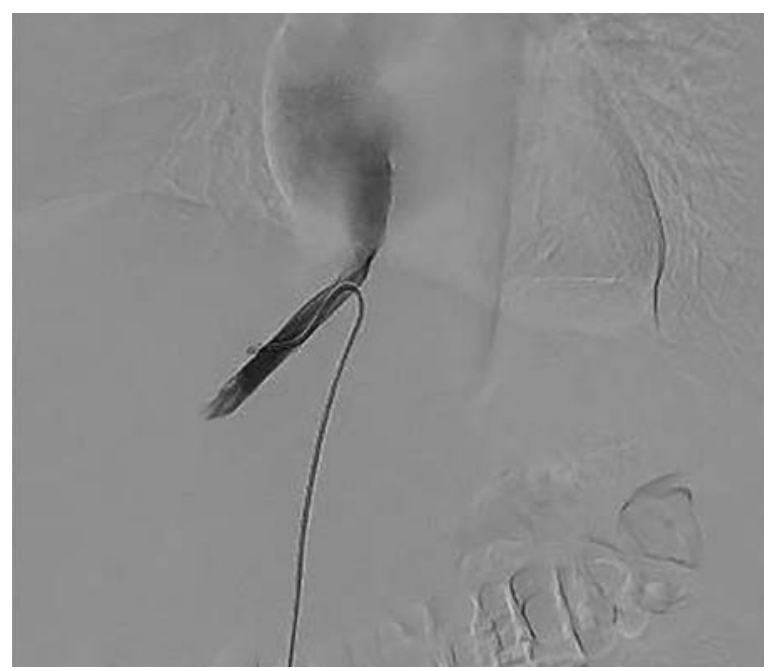

Figura 1. Catéter en vena suprahepática. 


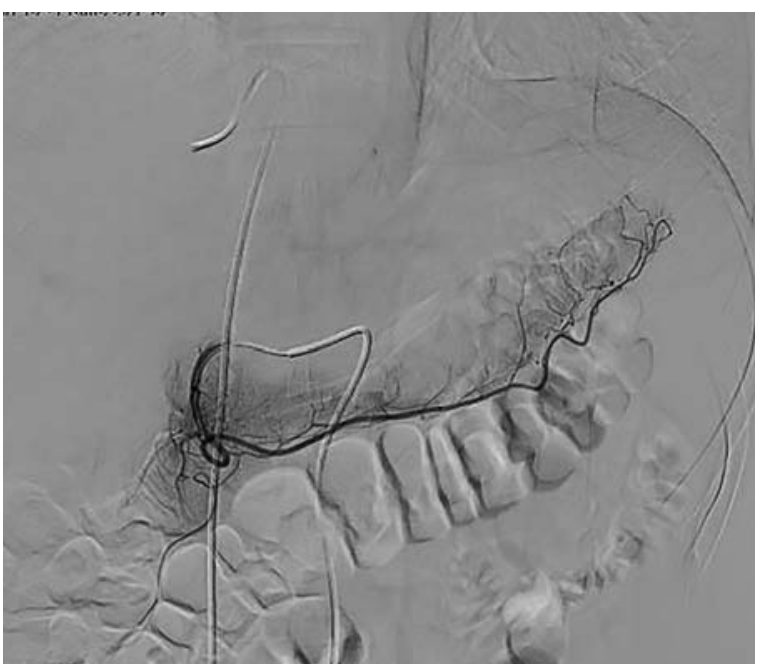

Figura 2. Microcatéter en arteria gastroduodenal.

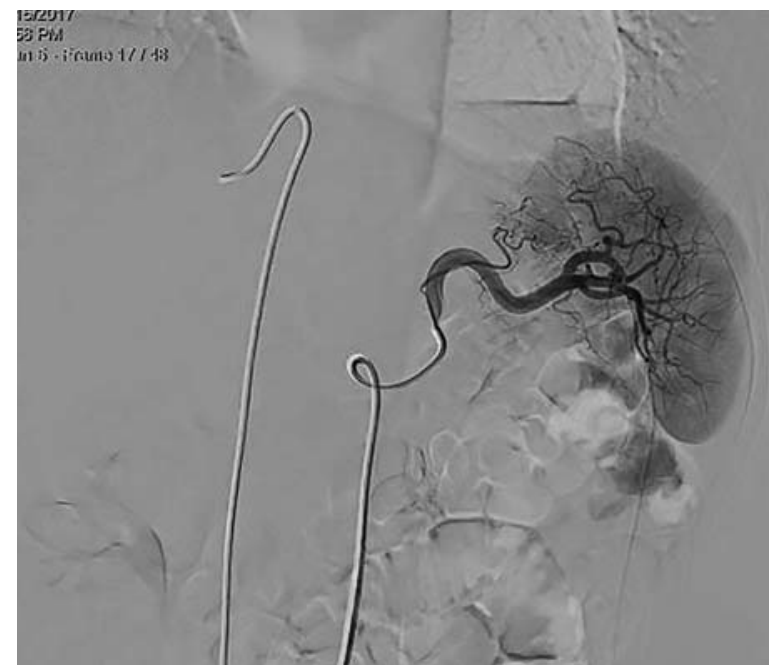

Figura 4. Catéter en arteria esplénica.

mesentérica superior y esplénica, cada una de las cuales tiene un área preferente de irrigación dentro del órgano ${ }^{4}$. Los insulinomas incrementan la secreción insulínica en respuesta a la infusión de calcio, mientras que las células beta normales no responden a dicho estímulo. Por otra parte, la infusión de calcio en la arteria hepática permite asimismo detectar áreas de hiperinsulinismo en el hígado que en ocasiones escapan a las pruebas morfológicas.

Al medir la respuesta insulínica en la vena hepática derecha tras la infusión de calcio en cada una de las tres arterias, podremos detectar el área susceptible de resección quirúrgica 5 . Dicha técnica, cuya sensibilidad y especificidad son superiores al 95\%, se utiliza conjuntamente con la angiografía de las tres arterias principales de la irrigación pancreática (gastroduodenal, esplénica y mesentérica superior), que puede detectar focos de hipervascularización sugestivos de patología tumoral hasta en un $75 \%$ de los casos.

La cuantificación de insulina plasmática se realizó por electroquimioluminiscencia (sistema Cobas 6000/ Módulo e601 - Roche Diagnostics).

Nuestro objetivo es demostrar la eficacia y sensibilidad de la técnica angiográfica utilizada y para ello presentamos a dos pacientes que fueron estudiadas en nuestros Servicios, las cuales, luego de confirmarse niveles de insulinemia inapropiadamente elevados durante episodios de hipoglicemia es-

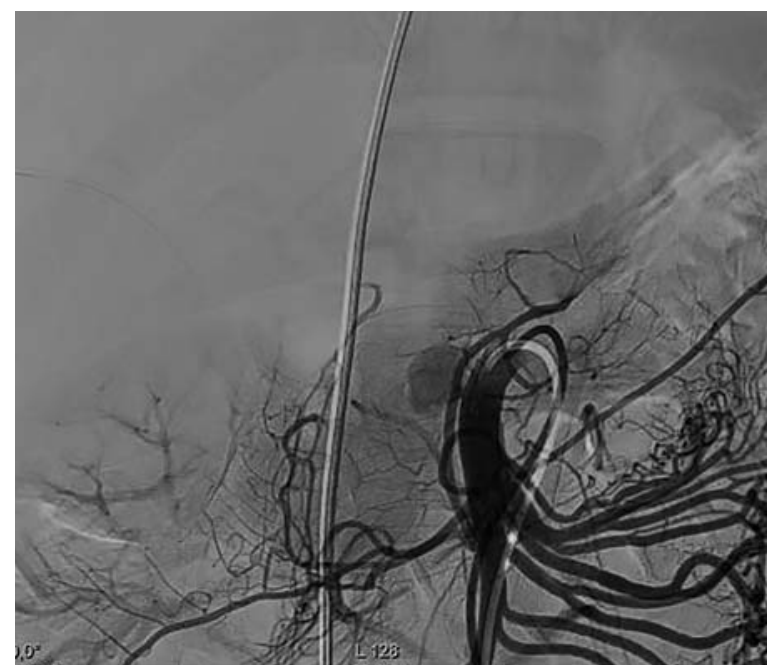

Figura 3. Catéter en arteria mesentérica.

pontáneos y con estudios imagenológicos no invasivos negativos, fueron sometidas a un estudio angiográfico y de estimulación intraarterial selectiva con calcio, con medición de insulinemias en vena suprahepática derecha, el que permite localizar con certeza el tumor y realizar una cirugía curativa.

\section{Técnica}

Se cateterizó vía femoral arterial con catéter cobra 5 Fr con progresión de un microcatéter de 2,4 Fr Progreat (Terumo Interventional Systems) de manera coaxial, además se introdujo por vena femoral un catéter Simmons 5 Fr, situando el catéter venoso en vena suprahepática (Figura 1) para recolección de muestras de insulinemias.

Con el catéter arterial se estimula selectivamente, con gluconato de calcio $(0,025 \mathrm{mEq}$ de $\mathrm{Ca} / \mathrm{kg}$ de peso), en las arterias que irrigan la cabeza pancreática (arteria gastroduodenal) (Figura 2) y mesentérica superior (Figura 3) y el cuerpo y cola (arteria esplénica) del páncreas (Figura 4).

Con el catéter venoso se recogen muestras de sangre para medición de insulinemia basales y a los 30, 60 y 120 s posteriores a la administración de calcio.

El procedimiento se realizó con soporte del servicio de anestesia, monitorización ECG, TA y medición de glucemia capilar, deteniendo la prueba ante la sospecha de signos de alarma.

\section{CASO CLÍNICO 1}

Paciente de sexo femenino de 25 años, sin antecedentes mórbidos de importancia, que comienza 3 años antes con cuadros de astenia, somnolencia, mareos, sudoración y temblores finos en extremidades que eran superados bebiendo bebidas dulces. La sintomatología evolucionó en forma progresiva, presentando posteriormente períodos de pérdida transitoria de conciencia, asociado al ejercicio extremo ya que la paciente pertenece a fuerzas de seguridad. Inicialmente se diagnosticó estrés y trastorno emocional. En uno de los episodios pseudocomiciales la paciente es hospitalizada en centro asistencial de la unidad donde estaba apostada, evaluada por diferentes especialidades médicas y luego de 30 días de internación sin diagnóstico de certeza, es derivada al Hospital Naval Dr. Pedro Mallo para estudio. Al examen físico destaca una glicemia de $34 \mathrm{mg} / \mathrm{dl}$, planteándose como primera posibilidad diagnóstica un insulinoma. 
ARTERIA ESPLENICA PROXIMAL

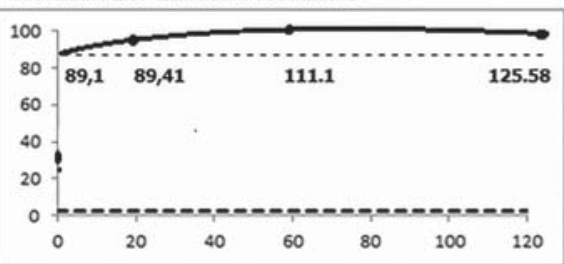

\section{ARTERIA GASTRODUODENAL}

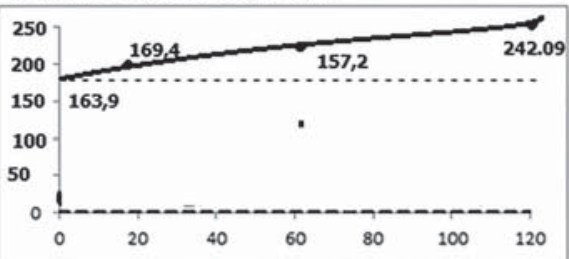

ARTERIA MESENTERICA

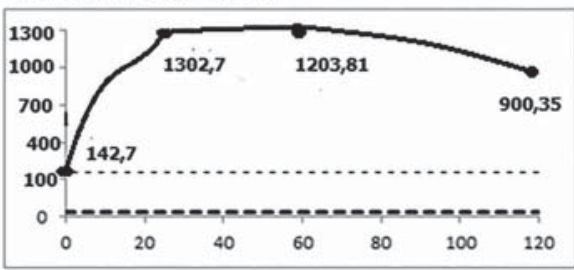

\section{ARTERIA ESPLENICA DISTAL}

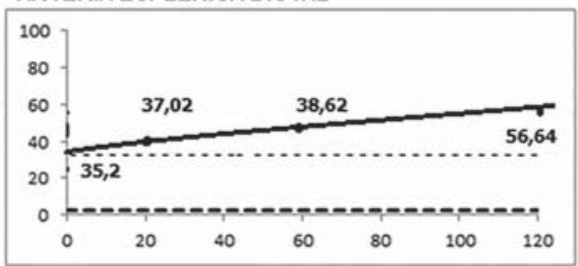

ARTERIA HEPATICA IZQUIERDA

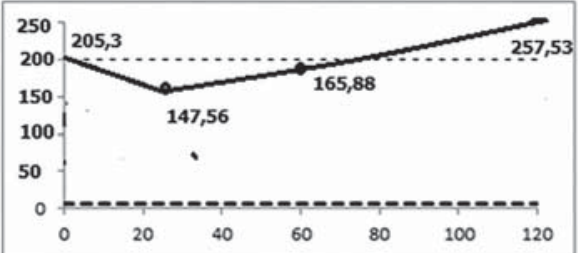

Figura 5. Valores de insulinemia en relación al tiempo, luego de estimulación arterial selectiva con calcio en el caso 1.

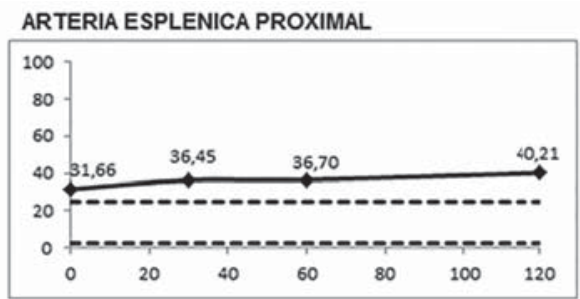

ARTERIA GASTRODUODENAL

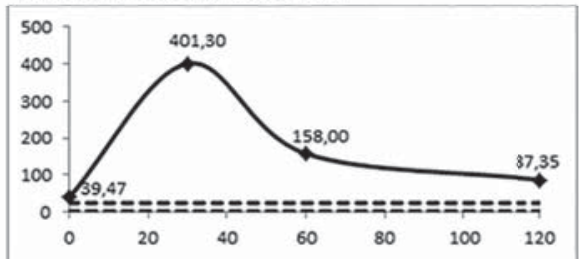

ARTERIA MESENTERICA

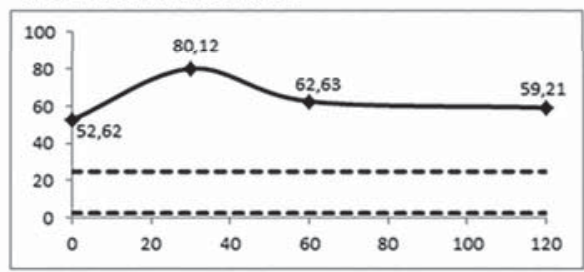

\section{ARTERIA ESPLENICA DISTAL}

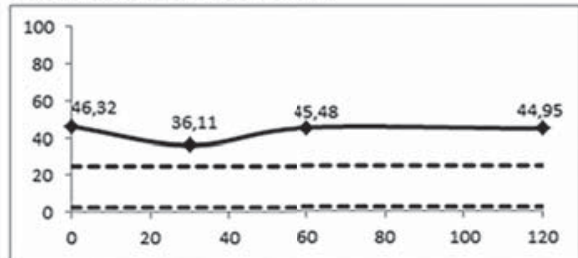

ARTERIA HEPATICA IZQUIERDA

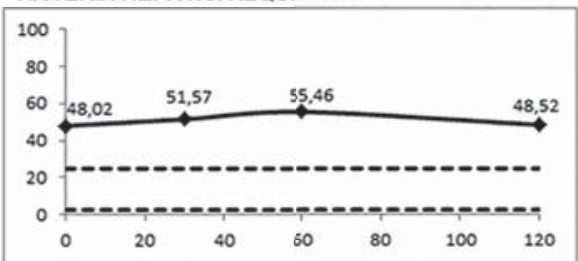

Figura 6. Valores de insulinemia en relación al tiempo, luego de estimulación arterial selectiva con calcio en el caso 2.

Se realizó estudio de localización con TC abdominal con resultados negativo y RM que informa lesión hiperintensa en cuerpo de páncreas. Se realizó angiografía pancreática que mostró una lesión hipervascularizada en el territorio de irrigación de la arteria pancreático duodenal inferior en topografía que corresponde a la cabeza del páncreas. Con este resultado, y para precisar la funcionalidad de la lesión encontrada, se sometió a test de estimulación con calcio de acuerdo al protocolo descrito por Doppman ${ }^{6}$.
Los resultados (Figura 5) concuerdan con la imagen obtenida en la angiografía y sugieren la presencia de un insulinoma localizado en la cabeza del páncreas. Fue sometida a cirugía, donde se palpó una lesión en cara anterior de la cabeza de aproximadamente 1,5 por 2 centímetros de diámetro, enucleándose el tumor. La biopsia rápida fue compatible con un tumor neuroendocrino del páncreas confirmándose posteriormente el diagnóstico histológico definitivo de insulinoma. 


\section{CASO CLÍNICO 2}

Paciente de sexo femenino de 26 años, sin antecedentes de importancia. Comienza con astenia, decaimiento y sudoración fría nocturna desde hace 1 año. Mantuvo glicemias en límite bajo a pesar hacer dieta con colaciones cada 3 horas.

Se realizó test de ayuno corto, a las 4 h glicemia de $39 \mathrm{mg} / \mathrm{dl}$, con insulinemia de 7,8 $\mu \mathrm{UI} / \mathrm{ml}$. Se indica TC de abdomen y RM de abdomen, que no logran identificar el tumor pancreático. El Servicio de Endocrinología del Hospital Privado de Rosario decide derivar al Servicio de Hemodinamia para realización de arteriografía selectiva pancreática con test de estimulación con calcio, con los siguientes resultados:

\section{DISCUSIÓN}

La localización de insulinomas con métodos no invasivos siempre ha tenido un bajo rendimiento. La TC alcanza una sensibilidad cercana al 40\%. La resonancia nuclear magnética ha reportado éxito en la localización de insulinomas en $60 \%{ }^{2}$. La medicina nuclear en este particular caso es poco útil. El octreotide, análogo de somatostatina, marcado con In-111, y que une a receptores de esta, tiene un rendimiento no superior al $50 \%$, por la escasez de receptores para somatostatina en este tumor ${ }^{5}$. Las células tumorales de un insulinoma tienen una respuesta exagerada a la estimulación con calcio. Sabemos que el calcio intracelular media en forma fisiológica la exocitosis de insulina por la movilización de los gránulos preformados de esta en la célula beta. En las células tumorales, esta respuesta está expresada por una sobre respuesta de las células tumorales al incremento en la concentración de calcio citosólico.

\section{BIBLIOGRAFÍA}

1. Service FJ, Mc Mahon MM, O'Brien PC, Ballard DJ. Functioning insulinoma incidence, recurrence and long term survival of patients: a 60 years study. Mayo Clin Proc 1991;66:711-9.

2. Machado MC, daCunha JE, Jukemura J et al. Insulinoma: diagnostic strategies and surgical treatment. A 22 years experience. Hepatogastroenterology 2001;48:854-8

3. Rosch T, Lightdale CJ, Botet JF, Boyce GA, Sivak MV Jr, Yasuda Ket al. Localization of pancreatic endocrine tumors by endoscopic ultrasonography. NEngl J Med 1992;326:1721-6.

4. Pereira, PL, Roche, AJ, Maier, GW, et al. Insulinoma and islet cell hyperplasia: Value of the calcium intra-arterial stimulation test when findings of other prospective studies are negative. Radiology 1998;206:703.

5. Meko JB, Doberty GM, Siegel BA, Norton JA. Evaluation of somatosta-
Doppman, en $1991^{6}$, fue el primero en describir la técnica de estimulación intraarterial con calcio para inducir una respuesta de secreción insulínica lo suficientemente potente para ser detectada en una muestra de sangre venosa temprana, de manera de evaluar el área de circulación pancreática y lograr localizar el tumor. Si al estimular a través de la arteria gastroduodenal o mesentérica superior con una concentración conocida de calcio se eleva la insulinemia más de 2 veces el basal, entonces el tumor debe estar en la cabeza del páncreas o en el cuello, como en el caso de nuestras pacientes. Si al estimular la arteria esplénica se produce el efecto descrito, el tumor lo debemos buscar en el cuerpo y cola pancreáticos. Este estímulo no afecta las células de los islotes normales porque están inhibidas por el tumor. El método tiene una sensibilidad reportada entre el 85 y $100 \%$ y una especificidad mayor de $90 \%$.

Este método ha sido también aplicado para el diagnóstico del fenómeno de hipoglicemia hiperinsulinémica en la infancia ${ }^{7}$ y también podría hacer sospechar una hipoglicemia facticia cuando no se produce respuesta frente al estímulo en ninguna de las tres arterias mencionadas ${ }^{8,9}$.

La estimulación intraarterial selectiva con calcio agrega solo unos minutos adicionales a la arteriografía, demorando el procedimiento total alrededor de sesenta minutos.

\section{CONCLUSIÓN}

La realización del test de estimulación con calcio bajo cateterismo selectivo de arterias que irrigan al páncreas representa un arma diagnóstica complementaria con alta sensibilidad y especificidad cuando el resto de las técnicas morfológicas (TC ,RM y ecografía endoscópica) no han conseguido la localización del insulinoma.

tin receptor scintigraphy for detection neuroendocrine tumors. Surgery 1996; 120:975-83.

6. Doppman JL, Miller DL, Chang R, Shawker TH, Gorden P, Norton JA. Insulinomas: localization with selective intraarterial injection of calcium. Radiology 1991;178:237-41.

7. Abernethy LJ, Davidson DC, Lamont GL, Shepherd RM, Dunne MJ. Intraarterial calcium stimulation test in the investigation of hyperinsulinaemic hypoglycaemia. Arch Dis Child 1998;78:359-63.

8. Palomares R, Zurera L, Gálvez MA, Tofe S, Canis M, Benito P. Utility of arteriography with selective arterial calcium injection for the diagnosis of insulinoma. Med Clin (Bar) 2002;119:568-70.

9. O'Shea D, Rohrer-Therus AW, Lynn JA, Jackson JE, Bloom JR. Localization of insulinomas by selective intra-arterial calcium injection. J Clin Endocrinol Metab 1996:81:1623-7. 